KOINONIA. Revista Arbitrada Interdisciplinaria de Ciencias de la Educación, Turismo, Ciencias Sociales y Económica, Ciencias del Agro y Mar y Ciencias Exactas y

aplicadas. Año IV. Vol IV. N7. Enero - Junio 2019. Hecho el depósito de Ley: FA2016000010 ISSN: 2542-3088

FUNDACIÓN KOINONIA (F.K). Santa Ana de Coro. Venezuela.

Nicolás Pablo Barrientos Oradini; Luis Araya Castillo; Victor Alan Veloso Salazar;Claudia Marcela Herrera Ciudad

\title{
Optimismo disposicional en docentes de universidades privadas en región metropolitana en Chile
}

\section{Dispositional optimism in teachers from private universities in the metropolitan region in Chile}

\author{
Nicolás Pablo Barrientos Oradini \\ nbarrientoso@docente.uss.cl \\ Universidad San Sebastián \\ Chile \\ https://orcid.org/0000-0002-5854-9501 \\ Luis Araya Castillo \\ luis.arayac@uautonoma.cl \\ Universidad Autónoma de Chile \\ Chile \\ https://orcid.org/0000-0001-7574-3907 \\ Victor Alan Veloso Salazar \\ vvelosoorcid@gmail.com \\ Universidad Miguel de Cervantes \\ Chile \\ https://orcid.org/0000-0001-8714-4485 \\ Claudia Marcela Herrera Ciudad \\ cherreraorcid@gmail.com \\ Universidad Miguel de Cervantes \\ Chile \\ https://orcid.org/0000-0002-4010-183X
}

Recibido: 10 de octubre del 2018

Aprobado: 09 de diciembre del 2018 


\title{
RESUMEN
}

El presente artículo se propuso analizar el optimismo disposicional en docentes que laboran en universidades privadas de la región metropolitana en Chile. Se realizó una revisión de las teorías relativas a la variable para dar cumplimiento a dicho propósito. Metodológicamente, la investigación se categorizó como descriptiva, con un diseño de campo, transeccional y no experimental. La población estuvo compuesta por los docentes que dictan clases en niveles de maestría en universidades privadas en la región metropolitana de Chile, seleccionada de forma intencional y representada por treinta y un (31) unidades informantes. Para la recolección de los datos se aplicó un cuestionario, conformado por seis (6) ítems. Para el análisis de los mismos se hizo uso de la estadística descriptiva. Los resultados permitieron visualizar una muy alta percepción de optimismo en los docentes, aunque igualmente se percibió una percepción moderada de pesimismo, infiriéndose que los docentes universitarios perciben que los objetivos que se proponen son alcanzables, mostrándose perseverantes al logro de los mismos. Las recomendaciones se enfocaron a proponer evaluaciones y consultoría psicológica periódicas a los docentes, orientada a diagnosticar a tiempo alguna actitud desfavorable para minimizar el pesimismo moderado registrado.

Descriptores: Actitud; propósito; docente; evaluación del docente; educación superior.

\begin{abstract}
The present article intends to show the results of the research that was oriented to analyze the optimism and the practices in teachers who work in private universities. A review of the theories related to the variable was carried out to fulfill said purpose. Methodologically, the research was categorized as descriptive, with a field design, transectional and non-experimental. The population was composed of teachers who teach classes at master's levels in private universities, selected intentionally and representative by thirty-one (31) reporting units. A questionnaire was applied, consisting of six (6) items, analyzing the results applying descriptive statistics. The results allowed to visualize a very high perception of optimism in the cases, although a moderate perception of pessimism was also perceived. The recommendations focused on proposing evaluations and psychological counseling, as well as the guidance of a doctor, guided the diagnosis of an unfavorable attitude.
\end{abstract}

Descriptors: Attitude; purpose; teacher; teacher evaluation; higher education. 


\section{INTRODUCCIÓN}

El aspecto que sin duda mantiene una alta relevancia en Latinoamérica dentro de los contextos del debate educativo ha sido lo referente a la remuneración de los docentes. (Londoño, 2007) Manteniendo la percepción general de que la misma es baja, llegando hasta niveles que algunos lo pudieran categorizar como indignos, y que ese desfase salarial ha sido el mayor obstáculo en la captación de un cuerpo docente motivado y de gran competencia, de allí que las remuneraciones inadecuadas resultan en la falta de motivación, siendo una dificultad para una educación de calidad.

De acuerdo con la situación actual no solo en Latinoamérica, sino la observada en la estructura remunerativa en las universidades privadas en Chile, más los desafíos que rodean a los docentes universitarios, se presume que se ha dificultado en el docente el establecimiento de vínculos con otros, sin que al mismo tiempo se perciba a sí mismo satisfecho o viva en un razonable equilibrio emocional. Este equilibrio es determinante según lo expresa Immordino-Yang y Damasio (2007), quién sostiene que "para educar bien hay que sentirse bien" (p.47), de forma que los procesos académicos tengan resultados significativos.

En otra perspectiva, Seligman (2008) señala que la labor docente es para muchos considerada altamente satisfactoria, y vista como un apostolado voluntario, lo cual genera en las personas diversos niveles y formas de bienestar psicológico y felicidad; es decir, el lado opuesto del estrés y el síndrome de burnout o "estar quemado por el trabajo" (p.87), que han sido estudiado por la psicología positiva.

Se torna de gran relevancia el hecho de asumir que el docente universitario debería poseer expectativas favorables hacia su entorno, ya que son aspectos que debe influir en la forma en que transmite el saber cognitivo a sus estudiantes o futuros profesionales. De no ser así, podría generar ansiedad, malestar, insatisfacción, hasta bajo rendimiento académico por parte de ellos al sentir una disminución en el estado de felicidad y un descenso en el rendimiento o desempeño en la labor de sus docentes, lo cual pudiera generar una cadena de emociones negativas. 
En este sentido, el propósito del estudio y que mediante el presente artículo se persiguió fue analizar el optimismo disposicional en los docentes.

\section{DESARROLLO}

\section{Optimismo Disposicional}

El estudio del optimismo se corresponde a los estudios Martínez-Correa, Reyes y González (2006), quienes en su teoría introducen una manera de dar explicación a las diferentes respuestas de afrontar dichos eventos vistos como negativos que le ocurren a las personas. Postulando la existencia de dos estilos explicativos: el optimista y el pesimista.

Costa-Requena, G., Cantarell-Aixendri, M., Parramon-Puig, G., y Serón-Micas, D., (2014), describen el optimismo disposicional como la expectativa o creencia generalizada que mantiene una persona que ocurrirán acontecimientos positivo en el futuro. Así, se acepta según los autores como el comportamiento conducido por ciertas expectativas y creencias que siempre ocurren situaciones y eventos positivos en la vida.

Estas definiciones coincide igualmente con lo manifestado por Chico (2002), para quién el optimismo disposicional se da cuando surgen dificultades, las expectativas favorables incrementan los esfuerzos de las personas para alcanzar los objetivos, y las expectativas desfavorables reducen tales esfuerzos, a veces hasta el punto de desentenderse totalmente de la tarea.

El optimismo disposicional y en general las creencias optimistas han sido estrechamente relacionados con un mejor ajuste y afrontamiento ante diversas situaciones consideradas estresantes, en la que la persona obtiene mejores resultados si ve como alcanzable la meta y evalúa los resultados de éxito como más probables que los negativos para triunfar (El-Anzi, 2005; Londoño, 2009).

Parece ser un rasgo estable a través de la vida, aunque no hay suficiente literatura al respecto; por ahora puede decirse que tiende a ser estable en el tiempo y a través de 
las diferentes situaciones, que corresponde a una cierta tendencia de las personas optimistas al considerar los eventos y situaciones negativas como eventuales y no permanentes causados por eventos externas a la persona, (Scheier, et al, 2001), contrariamente a la actitud de pesimista que revela las situaciones negativas como generadas por una condición individual y que generalmente es duradera. (Seligman, 2003).

Incluso las observaciones que se han evidenciado muestran una asociación con la motivación, la salud y las estrategias de afrontamiento de estados de estrés (Scheier y Carver 2014; Solberg Nes y Segerstrom, 2009). Específicamente y con relación a la motivación, los estudios muestran que las personas que mantienen un nivel alto de optimismo se caracterizan porque realizan un esfuerzo mayor en el cumplimiento de las metas, por otro lado aquellas personas que muestran pesimismo, generalmente tienden a un menor involucramiento y tienden a realizar un esfuerzo menor en sus acciones.

Partiendo de las concepciones de los autores anteriormente citados sobre dicha variable, se entiende el mismo como la expectativa o creencia estable de que en la vida ocurrirán cosas positivas, al asumir que el futuro depara éxito y no fracasos, logrando en los individuos ajustarse o afrontar las situaciones que puede considerar estresantes en la vida y así obtener resultados beneficiosos al ver metas posibles de alcanzar.

\section{Dimensiones del Optimismo Disposicional}

\section{Optimismo}

Desde la visión positiva de la salud se ha estudiado y definido el optimismo como la preferencia de una persona a ser motivada por la creencia que los resultados deseados son fácilmente alcanzables, relacionado con efectos positivos sobre la salud psicológica y física (Brannon y Feist, 2001); así mismo, ha sido identificado como factor determinante de la calidad de vida tanto en personas enfermas como personas sanas. El optimismo se constituye desde la habilidad del sujeto para interpretar y recordar las experiencias con un matiz positivo. Si se puede hacer eso de manera regular confiando 
en la capacidad para controlar los estados de ánimo, todas las ventajas que emanan de una visión optimista de la vida estarán a alcance, ya que de igual forma las demás personas perciben y responden de manera positiva a la actitud de los optimistas, dándoles preferencias en el trabajo, en el amor, hasta en el juego, siendo a menudo acompañada de éxito (Velasco, Londoño, y Castañeda (2014).

En otra perspectiva, Seligman (2003) refiere que significa tener una fuerte expectativa de que, en general, las cosas irán bien a pesar de los contratiempos o las frustraciones. Los optimistas tienen la tendencia a reaccionar de manera activa y esperanzada, desarrollando un plan de acción por sí mismos o buscando ayuda, ya que consideran que los contratiempos no son imposibles de resolver.

El optimismo entonces, es entendido, luego de haber revisado las concepciones anteriores, como la creencia de los individuos al visualizar como alcanzables los objetivos que se proponen, mostrándose perseverantes, motivadas y positivas al logro de los mismos. Además, es la tendencia a creer que experimentarán buenos resultados a pesar de los contratiempos que puedan presentarse. (Remor, Amorós y Carrobles 2006).

\section{Pesimismo}

Se entiende a una actitud de pesimismo cuando se muestra un comportamiento que tiende a desarrollar un juicio sobre las realidades con acepciones desfavorables de en ser concretadas. En esa perspectiva y en opinión de Seligman (2008), dicha comprensión describen a las personas pesimistas y los categorizan con el grupo que regularmente consideran que las situaciones y eventos que son de una forma desagradable o que producen ciertos momentos dolorosos, duren un tiempo muy largo. Estas personas casi siempre subestiman la capacidad para realizar cambios y atribuyendo los fracasos a sus propias personas. (Londoño, Hernández, Alejo y Pulido, 2013). 
Además, se ha notado que el pesimismo da orígenes a procesos depresivos, produciendo una autoestima baja e incluso comportamientos de ansiedad (Alpízar y Salas 2010), comportamiento que tiende a impedir la acción de enfrentar los obstáculos, desistiendo ante ellos, incluso cuando es posible lograra un éxito. En esa misma perspectiva, se denota que los jóvenes que suelen mostrar una actitud de pesimismo, generalmente registran efectos negativos que se expresan en desesperanza, un alto nivel de ansiedad, que en algunas ocasiones los conducen al consumo de sustancias (Lemola et al., 2010).

El pesimismo es entendido entonces, como la creencia de que el futuro deparará cosas negativas, atribuyéndose la persona las causas por las que fallaron en el logro de sus metas propuestas. Además, las personas pesimistas pueden manifestar depresión, angustia o ansiedad, impidiendo superar los obstáculos que se les puedan presentar.

\section{Indicadores del Optimismo Disposicional}

\section{Visión positiva hacia el futuro}

Hartmann, Chneider y Emrich, (2003), afirma que "la fuerza de una tendencia a actuar en determinada forma depende de que el acto esté seguido por un resultado determinado y de lo atractivo de ese resultado para el individuo" (p.68). De acuerdo a los autores es posible conectar la expectativa con la conducta, originando la motivación, la cual es definida por el autor como "un proceso que controla la elección de las formas alternativas de control sobre la actividad voluntaria. Desde su punto de vista, la mayoría de las conductas están bajo el control voluntario de la persona y motivadas por ésta" (Londoño, 2009: 57).

De allí, que se entiende al optimismo como la tendencia a esperar que en el futuro ocurran eventos positivos. Según Marrero y Carballeira (2010) tales expectativas se consideran, además, como disposiciones que permanecen estables, esto es, rasgos. Se dice que las personas optimistas tienden a ser más esperanzadas o perseverantes, tener mejor autoestima y generar acciones más exitosas; mantienen más alerta sus 
defensas inmunológicas, se muestran más activas frente a las dificultades, toman más decisiones así como adoptan mayor cantidad de medidas para crearse una red de apoyo afectivo y social (Morales, et al .(2011).

Al analizar los postulados de los autores anteriormente citados, se puede inferir que la visión positiva hacia el futuro significa la expectativa de obtener resultados favorables en la vida de los individuos, mostrando autoestima y generando vías de logro de objetivos más exitosas al tomar mejores decisiones frente a contingencias presentadas.

\section{Visión negativa hacia el futuro}

De acuerdo a los planteamientos expuestos por Vera (2006) se comprende el pesimismo como la expectativa de que sucedan acontecimientos desfavorables. Los estudios realizados sobre este tema, han encontrado que las personas pesimistas mantienen una relación directa con el empleo de estrategias de afrontamiento centradas en la emoción después de un fracaso (Peterson, 2000).

Las personas pesimistas frente a cualquier amenaza creen estar indefensas. Al tratar de explicar las causas de las situaciones en las que se encuentran, tienden a hacerlo con un estilo atribucional penetrante, permanente y personal, lo cual genera un sentimiento de abandono; esperan encontrarse siempre desamparadas. Es decir, cuando se encuentren en situaciones similares volverán a sentirse indefensas (CostaRequena et al. 2014).

Básicamente, cuando surgen dificultades, las expectativas desfavorables reducen los esfuerzos de las personas para alcanzar objetivos. Según Vera (2006) la visión negativa del futuro supone que cuando la persona hace proyectos de gran alcance, está anticipando que sus dificultades actuales continuarán indefinidamente, espera penas, frustraciones y privaciones interminables.

En relación a los autores citados, se entiende la visión negativa hacia el futuro como la expectativa de que sucedan situaciones desfavorables, anticipando que el logro de sus 
metas se convertirán en un fracaso, reduciendo de esta forma sus esfuerzos para plantearse nuevos objetivos en el futuro.

\section{MATRIZ METODOLÓGICA}

El estudio estuvo enmarcado dentro de los criterios de una investigación de tipo descriptiva, de manera que buscó conocer las características en relación a la variable de la población que fue objeto de estudio conformada por los docentes de universidades privadas, en este caso, el bienestar psicológico y el optimismo disposicional en docentes de instituciones universitarias del sector privado, observando las variables en el contexto.

En este sentido, Hernández, Fernández y Baptista (2010) definen el tipo de investigación descriptiva como "la búsqueda específica de propiedades, características y rasgos importantes de cualquier fenómeno que se analice" (p. 119), describiendo tendencias de un grupo o población. De igual manera, se caracteriza por ser de campo, transeccional o transversal, y no experimental, basado en los supuestos de Hurtado (2010), Tamayo y Tamayo (2007).

El propósito del estudio estuvo dirigido a describir la variable optimismo disposicional en los docentes universitarios de universidades privadas, al observar los hechos y eventos en un contexto real para un análisis posterior, desprendiéndose reflexiones por parte del investigador. Es por ello que la muestra estuvo conformada por los docentes que imparten clases a nivel de Maestría, siendo representada con un total de treinta y un (31) unidades informantes en total.

De esta manera, se seleccionó la administración de un cuestionario como instrumento, haciendo posible la recolección de datos detalladamente, en relación con los indicadores por dimensión de la variable de estudio. Dicho esto, el presente cuestionario está conformado por un conjunto de seis (6) premisas, realizadas de acuerdo a cada uno de los dos (2) indicadores que representan la dimensión de la 
variable objeto de estudio, siendo las mismas la visión positiva hacia el futuro y la visión negativa hacia el futuro.

\section{RESULTADOS}

A continuación, se presenta el análisis de los resultados obtenidos en los cuestionarios aplicados a la población total de estudio, lo cual permitirá dar respuesta los objetivos específicos al interpretar las puntuaciones de cada indicador junto a sus respectivos ítems. En este sentido, se expresarán las puntuaciones de los sujetos en determinadas tablas para así lograr un diagnóstico certero en cuanto a la realidad de dicha población, al aplicar la estadística descriptiva mediante matrices de doble entrada utilizando el programa computarizado de Excel y calculando las frecuencias relativa y absoluta para ubicar la puntuación de la media tomando como referencia el siguiente baremo de interpretación:

Tabla 1. Escala de interpretación de medias

\begin{tabular}{cc}
\hline Categorías & Rangos \\
\hline Muy Alta & $4,21-5$ \\
\hline Alta & $3,41-4,2$ \\
\hline Moderada & $2,61-3,4$ \\
\hline Baja & $1,81-2,6$ \\
\hline Muy Baja & $1-1,8$ \\
\hline Fuente: elaboración propia (2018)
\end{tabular}

Tabla 2. Dimensión: Optimismo

\begin{tabular}{|c|c|c|c|c|c|c|c|c|}
\hline \multicolumn{9}{|c|}{ Alternativas de Respuestas } \\
\hline & Ítem & Siempre & $\begin{array}{c}\text { Casi } \\
\text { Siempre }\end{array}$ & $\begin{array}{c}\text { Algunas } \\
\text { Veces }\end{array}$ & $\begin{array}{c}\text { Casi } \\
\text { Nunca }\end{array}$ & Nunca & \multicolumn{2}{|c|}{ Promedio } \\
\hline \multirow{4}{*}{$\begin{array}{c}\text { Visión } \\
\text { Positiva } \\
\text { del } \\
\text { Futuro }\end{array}$} & & $\%$ & $\%$ & $\%$ & $\%$ & $\%$ & Media & Categoría \\
\hline & 1 & 54,83 & 25,80 & 19,35 & 0 & 0 & \multirow{3}{*}{4,53} & \multirow{3}{*}{ Muy Alta } \\
\hline & 2 & 74,19 & 22,58 & 3,22 & 0 & 0 & & \\
\hline & 3 & 64,51 & 25,80 & 9,67 & 0 & 0 & & \\
\hline
\end{tabular}

Fuente: Elaboración propia (2018) 
Como se puede apreciar, para el indicador Visión positiva hacia el futuro, 23 de los encuestados que representan un $74,19 \%$ de la población, contestaron que siempre se muestran frente a las dificultades como personas activas, así como se perciben a sí mismo esperanzados y perseverantes, tal y como 8 de ellos que son un $25,80 \%$ indican que casi siempre se perciben de esta manera, así como 6 sujetos representando un $19,35 \%$ respondieron que algunas veces es de este modo. En este sentido, el presente indicador logró obtener una puntuación de 4,53 sobre 5, ubicándose dentro de la categoría muy alta según la escala diseñada para interpretar las medias calculadas En este sentido, por medio de los promedios alcanzados por cada indicador de la dimensión Optimismo, se logró conocer que la misma alcanzó un puntaje de 4,53 de 5, lo cual según la escala diseñada para interpretar las medias calculadas, se ubica en una categoría muy alta, indicando que los docentes universitarios visualizan como alcanzables los objetivos que se proponen, mostrándose perseverantes al logro de los mismos, así como poseen la convicción que experimentarán buenos resultados a pesar de los contratiempos que puedan presentarse.

Tabla 3. Dimensión: Pesimismo.

\begin{tabular}{|c|c|c|c|c|c|c|c|c|}
\hline \multicolumn{9}{|c|}{ Alternativas de Respuestas } \\
\hline & Ítem & Siempre & $\begin{array}{c}\text { Casi } \\
\text { Siempre }\end{array}$ & $\begin{array}{c}\text { Algunas } \\
\text { Veces }\end{array}$ & $\begin{array}{c}\text { Casi } \\
\text { Nunca }\end{array}$ & Nunca & & medio \\
\hline \multirow{4}{*}{$\begin{array}{c}\text { Visión } \\
\text { Negativa } \\
\text { del } \\
\text { Futuro }\end{array}$} & & $\%$ & $\%$ & $\%$ & $\%$ & $\%$ & Media & Categoría \\
\hline & 4 & 9,67 & 58,06 & 29,03 & 3,225 & 0 & \multirow{3}{*}{2,74} & \multirow{3}{*}{ Moderada } \\
\hline & 5 & 3,22 & 16,12 & 41,93 & 22,58 & 16,12 & & \\
\hline & 6 & 3,22 & 3,22 & 9,67 & 38,70 & 45,16 & & \\
\hline
\end{tabular}

Fuente: Elaboración propia (2018)

Con respecto a la Tabla 3, para el indicador Visión negativa hacia el futuro, 18 docentes representando un $58,06 \%$ de la población contestaron que casi siempre esperan privaciones al trazar una nueva meta en su vida, tiene expectativas desfavorables en cuanto a los objetivos que se plantean y anticipan las dificultades que se les presentarán al realizar un proyecto, así como 14 de ellos que son un 45,16\% aseguran 
que nunca es de esta forma, 13 que representan un $41,93 \%$ indican que algunas veces es así, 12 de los mismos que son un 38,70\% respondieron casi nunca y, por último, 3 de los sujetos que son un $9,67 \%$ dijeron que siempre lo esperan, ubicando al indicador en una categoría moderada, al obtener 2,74 puntos de 5 .

De esta manera, mediante los promedios alcanzados por cada indicador de la dimensión Pesimismo, se logró conocer que la misma alcanzó un puntaje de 2,74 sobre 5 , lo cual según el baremo para la interpretación de la media, se ubica en una categoría moderada, indicando que los docentes universitarios poseen la creencia de que el futuro deparará cosas negativas, atribuyéndose las causas por las que fallaron en el logro de sus metas propuestas, impidiendo superar los obstáculos que se les puedan presentar.

\section{CONCLUSIONES}

Resulta importante recalcar que de acuerdo a los resultados obtenidos por los docentes universitarios, se visualiza una muy alta percepción de optimismo en ellos, aunque también una moderada percepción de pesimismo; aun siendo menor la categoría del pesimismo con respecto a la del optimismo, no se puede evitar mencionar. En este sentido, se logra inferir que la labor docente es capaz de generar muy altos niveles de optimismo por la gratificación que contiene esta labor per sé, conllevando a sentir una fuerte sensación de logros adquiridos a través del éxito de sus estudiantes, reconocimientos académicos o superación profesional.

Sin embargo, en relación a lo anterior, así como se sienten optimistas en diversos aspectos, de manera moderada no descartan sus sentimientos de pesimismo, al presentar algunas veces una visión negativa hacia el futuro, quizás por diferentes limitaciones externas a sus voluntades, ya sea preocupación por la situación actual venezolana, por la imposibilidad de obtener los recursos materiales que desean debido a sus salarios o la desmotivación y frustración proyectada por parte de sus estudiantes, así como los constantes absentismos. 


\section{Reflexiones finales}

Luego de haber presentado un análisis conclusivo desde los resultados del estudio, los investigadores pasan a exponer algunas reflexiones orientadas a los docentes que se constituyó en población estudiada, así como al entorno institucional educativo y investigadores interesados en desarrollar y continuar investigaciones en la temática abordada, todo ello con la finalidad de generar aportes positivos para el desarrollo personal, social y laboral de la comunidad docente.

Es relevante que el Optimismo en docentes de las instituciones, se recomienda a las instituciones educativas fortalecer las capacidades para el desarrollo de los sentimientos de perseverancia y superación, en el diseño de ofertas académicas relativas en asignaturas, programas y estrategias innovadoras que desarrollen sus competencias intelectual y profesional.

De igual manera, es relevante que los docentes, den continuidad a la proyección de logros de forma que se mantengan activos y permitan plantearse nuevos retos favorables para su crecimiento profesional y académico.

Sin embargo, la presencia aunque moderada del nivel de Pesimismo en docentes, es importante considerar programas que orientados a afrontar situaciones que ante una contingencia presentada en el desempeño de su rol, que les cause una disminución de motivación al logro, abandonar cualquier tipo de pensamiento negativo o sentimiento de indefensión de forma que se evite proyectarlo a los alumnos que los consideran modelos a seguir.

En relación a lo anteriormente expresado es importante destacar lo expresado por Gutiérrez; Rodríguez e Isea (2018):

La emoción forma parte de cada persona y desempeña un papel muy importante en la manera de actuar, en el aprendizaje, creatividad e interacción social, las emociones y los estados de ánimos están asociados a las experiencias que vive una persona, la acción que ejecuta un individuo ante una determinada situación es lo que se conoce como emoción y el sentimiento en el que se sumerge la persona es el estado de ánimo, por lo tanto, en la formación universitaria, es importante saber diferenciarlas y 
tenerlas presente para evitar problemas que generen desacuerdos cuando se implementa una transformación curricular, pues cuando se involucra a todos los actores educativos en el cambio, se les escucha, se les toma en cuenta en todas o en la mayoría de las decisiones, donde sus opiniones son valoradas; éstos sentirán que sus voces son escuchadas y por ende, sentirán que son parte importante del cambio curricular, pues ayudaron a construirlo para el beneficio de todos, y los problemas que se susciten durante la ejecución, no generarán angustias ni rechazo, pues podrán ser solventadas por todos como un equipo y no por un pequeño grupo que capitalice las tomas de decisiones (p. 244).

Para esto, es importante realizar evaluaciones y consultoría psicológica periódicamente a los docentes para diagnosticar a tiempo una actitud desfavorable, o bien, prevenir sentimientos de malestar y desmotivación que en el futuro ya no se puedan modificar o manejar.

\section{REFERENCIAS CONSULTADAS}

1. Alpízar, H., y Salas, D. (2010). El papel de las emociones positivas en el desarrollo de la Psicología Positiva. Revista Electrónica de estudiantes Esc. de psicología, 5(1), 65-83. Universidad de Costa Rica. Recuperado de http://www. dialnet.unirioja.es.

2. Brannon, L. y Feist, B. (2001). Psicología de la Salud. Madrid España: Thompson.

3. Chico E. (2002). Optimismo disposicional como predictor de estrategias de afrontamiento. Psicothema 14(3):544-50.

4. Costa-Requena, Gemma, Cantarell-Aixendri, M. Carmen, Parramon-Puig, Gemma, y Serón-Micas, Daniel. (2014). Optimismo disposicional y estrategias de afrontamiento en pacientes con trasplante renal. Nefrología (Madrid), 34(5), 605610. https://dx.doi.org/10.3265/Nefrologia.pre2014.Jun.1188.1.

5. El-Anzi, F. O. (2005). Academia achievement and its relationship with anxiety, self-esteem, optimism, and pessimism in Kuwaiti students. Social Behavior and Personality, 33 (1) 95-103.

6. Gutiérrez, N., Rodríguez, N., \& Isea, J. (2018). LA EMOCIONALIDAD FRENTE A LA INNOVACIÓN CURRICULAR. Revista Arbitrada Interdisciplinaria Koinonía, 
http://fundacionkoinonia.com.ve/ojs/index.php/revistakoinonia/article/view/61

7. Hartmann, U., Chneider, U., y Emrich, M. (2003). La búsqueda de la felicidad. Revista Mente y Cerebro, 4,78-83. Recuperado de http://dialnet. unirioja.es/servlet/articulo?codigo=2809082.

8. Hernández, R., Fernández, C., y Baptista, P. (2010). Metodología de la Investigación. México D.F.: Mcgraw-HILL / Interamericana Editores, S.A. de C.V.

9. Hurtado, J. (2010). El proyecto de investigación: "Comprensión holística de la metodología y la investigación". Venezuela: Ediciones Quiron.

10.Immordino-Yang, M. H., \& Damasio, A. (2007). We feel, therefore we learn: the relevance of affective and social neuroscience to education. Mind, Brain and Education, 1(1), 3-10.

11.Lemola, S.; Räikkönen, K.; Matthews, K.; Scheier, M.; Heinonen, K. (2010). A New Measure for a Dispositional for Optimism and Pessimis in Young Children, European Journal of personality, 24(1), 71-84.

12.Londoño, C. (2007). Construcción de un modelo cognitivo social integrado para la prevención del abuso de alcohol en universitarios bogotanos. Tesis de maestría, Facultad de Psicología, Universidad Nacional de Colombia.

13.Londoño, C., Hernández, L., Alejo, I., y Pulido, D. (2013). Diseño y validación de la Escala de Optimismo Disposicional/Pesimismo -EOP-. Universitas Psychologica, 1(12), 139-155. Recuperado de http://revistas.javeriana.edu.co/sitio/psychologica/sccs/articulo.php?id=701\&PHP SESSID=b40951e99064c3897f615e54a9 a950e0.

14. Londoño, C. (2009). Optimismo y salud positiva como predictores de la adaptación académica. Acta colombiana de psicología: 12(1), 95-107. Universidad Católica de Colombia. Recuperado de http://portalweb.ucatolica.edu.co/easyWeb2/files/23_3051_v12n1-art8.pdf.

15. Marrero. R., y Carballeira, M. (2010). El papel del optimismo y del apoyo social en el bienestar subjetivo. Salud mental, 33,39-46. Recuperado de http://www.artemisaenlinea.org. mx/acervo/pdf/salud_mental/El\%20papel\%20 del\%20optimismo.pdf. 
16. Martínez-Correa, A., Reyes A., González M., (2006). Optimismo/pesimismo disposicional y estrategias de afrontamiento del estrés. Psicothema 18(1), 66-72.

17. Morales, A. I., Arenas, M. D., Reig-Ferrer, A., Álvarez, F., Malek, T., Moledous, A., Gil, M. T., y Cotilla, E. M. (2011). Optimismo disposicional en pacientes en hemodiálisis y su influencia en el curso de la enfermedad. Nefrología, 31(2), 199205. http://dps.ua.es/es/documentos/pdf/2011/optimismodisposicional-pdf.

18. Peterson, Ch. (2000). The future of optimism. American Psychologist, 55(1), $44-$ 55.

19. Remor, E., Amorós, M., y Carrobles, J. (2006). El optimismo y la experiencia de ira en relación con el malestar físico. Anales de Psicología, 22(1), 3744. Recuperado de http://redalyc.uaemex.mx/redalyc/src/inicio/ArtPdfRed.jsp?iCve=16722105\&iCve Num=0.

20.Scheier, M. F., y Carver, C. S. (2014). Dispositional optimism. Trends in cognitive sciences, 18(6), 293-299. DOI: 10.1371/journal.pone.0097752.

21.Scheier, M. F., Carver, C. S. y Bridges, M. (2001).Optimism, pessimism, and psychological well-being. In E. C. Chang (Ed.), Optimism and pessimism: Implications for theory, research, and practice 189-216. Washington, DC: American Psychological Association.

22. Seligman, M. (2003). La Auténtica Felicidad. Barcelona, España: Byblos.

23.Seligman, M. (2008) Positive Health. Applied Psychology: An International Review, 57(1),3-18.

24.Solberg Nes, L., Evans, D. R. \& Segerstrom, S. C. (2009). Optimism and college retention: Mediation by motivation, performance, and adjustment. Journal of Applied Social Psychology, 39(7), 1887-1912.

25. Tamayo y Tamayo (2007). El proceso de la investigación científica. 4ta.ed. México: Limusa.

26. Velasco, M., Londoño, C., y Castañeda, I., (2014). Validación del cuestionario de optimismo disposicional usando la teoría de respuesta al ítem. Revista Diversitas- Perspectivas en psicología. 10(2), 275-292. 
KOINONIA. Revista Arbitrada Interdisciplinaria de Ciencias de la Educación, Turismo, Ciencias Sociales y Económica, Ciencias del Agro y Mar y Ciencias Exactas y aplicadas. Año IV. Vol IV. Nº . Enero - Junio 2019. Hecho el depósito de Ley: FA2016000010 ISSN: $2542-3088$

FUNDACIÓN KOINONIA (F.K). Santa Ana de Coro. Venezuela.

Nicolás Pablo Barrientos Oradini; Luis Araya Castillo; Victor Alan Veloso Salazar;Claudia Marcela Herrera Ciudad

27. Vera, B. (2006). Psicología Positiva: Una nueva forma de entender la psicología. Papeles del Psicólogo, 27(1), 3-8. Recuperado de http:// redalyc.uaemex.mx/src/inicio/ArtPdfRed. jsp?iCve=77827102.

C2019 por el autor. Este artículo es de acceso abierto y distribuido según los términos y condiciones de la licencia Creative Commons Attribution (http://creativecommons.org/licenses/by/4.0/). 